\title{
Palliative Care Guide
}

\author{
Loretta J Kaes*, Marjorie Risola, Lisa Williams, Mihail Davidovich, Gloria Horton, Lois Beatty, Linda Sue \\ Patron, Kristin Mancini, Regina Moschik and Pat Alfano
}

Director of Quality, Health Care Association of New Jersey, USA

Received: September 02, 2017; Published: September 13, 2017

*Corresponding author: Loretta J Kaes, Director of Quality, Improvement and Clinical Services, Health Care Association of New Jersey, New Jersey, USA

\begin{abstract}
When confronting chronic and/or serious illness many individuals wish to be active participants in determining the treatment and care provided to sustain optimal quality of life. As healthcare professionals, our goal is to facilitate a conversation with the individual and/or their family or other advocates that supports that person's life's journey and addresses their physical, social, spiritual, and emotional needs, as well as their values and cultural traditions. Through this discussion, we assist the person in using a systematic approach to identify and coordinate a plan of care to maintain quality of life.
\end{abstract}

Abbreviations: POLST: Providers Orders for Life Sustaining Treatment

\section{Introduction}

The acknowledgement of the complex needs surrounding chronic/serious illness and the importance and benefit of palliative care, led to palliative medicine becoming recognized in 2006 as a board-certified sub-specialty of internal medicine with specialized fellowships for physicians in the United States. Palliative care is a multidisciplinary approach to person-centered care, relying on input from pharmacists, nurses, chaplains, social workers, psychologists, and other allied health professionals in formulating a plan of care to help relieve suffering in all aspects of a seriously ill person's life. This multidisciplinary approach allows the palliative care team to address physical, emotional, spiritual and social concerns that arise with advanced illness [1-4].

Palliative care focuses on providing the individual with relief from the symptoms, pain, and physical and emotional stress of a serious or chronic illness whatever the diagnosis. Palliative care is provided by a team as an extra layer of support by healthcare, ancillary, and spiritual staff. It is appropriate at any age and at any stage in a serious illness and can be provided as the main goal of care or along with curative treatment. Palliative care can be provided across multiple settings in healthcare settings, in the person's home, or as part of community palliative care programs.

\section{Key Elements in a Palliative Care Program}

\section{Element One (Education)}

\section{Determine who will be the educators}

Select a "Champion" educated in the palliative care concept and effective in "having the conversation." This champion will educate other individuals who wish to be a part of the Palliative Care team.

\section{Advanced Care Planning Education- Leadership Roles}

A. Individuals who is engaging, enthusiastic and has demonstrated competency in the practice of palliative care.

B. Director of Nursing Services/Advanced Nurse Practitioner/Corporate or contracted provider.

C. Direct care staff

D. Director of Social Services

E. Rehabilitation therapist

F. Chaplain

G. Training specific to your healthcare environment

H. Acute care

I. Post-acute care

J. Hospice agency

K. Assisted living

L. Skilled nursing

M. Home care

N. Ensure individuals are sufficiently trained and comfortable in starting the conversation for advance care planning: Suggested times for the conversation may be
a) During care plan/family conference
b) MDS schedule - 5-day/14-day/30-day/60-day/ quarterly 
c) In assisted living - resident/responsible representative conference/6-month review

d) Following a significant change in condition

\section{Element Two}

Create an Advance Directive, along with the individual, that identifies a healthcare agent and stated goals of care

A. Advance care planning means the individual has determined what his/her personal goals, values, religious, cultural beliefs are and what defines their quality of life.

B. The conversation will include a discussion of the treatment(s) the individual may want to receive and what treatment to withhold.

C. This document will provide the health care professional and loved ones with valuable information to coordinate goals of care and discuss chronic disease exacerbation and possible palliative treatment at end of life to alleviate pain and suffering.

The POLST (Providers Orders for Life Sustaining Treatment) is an approach to end-of-life planning those states patients' wishes about medical treatments they want to receive and emphasizes:

A. Advance care planning conversations between patients, health care professionals and loved ones.

B. Informed shared decision-making between a patient and his/her health care professional about the treatment the patient would like to receive at the end of life.

C. Ensuring patient treatment wishes are honored. The decisions from these conversations may be documented as actionable medical orders on a POLST form.

A POLST form is an official Provider's Medical Order that ensures patients' treatment wishes are known and will be followed by health care professionals during a medical crisis when the patient cannot speak for him/herself. The POLST form may vary in appearance from state to state, but an officially signed form by a licensed provider will be honored across state lines by all levels of healthcare. Five Wishes originated in 1996 as a Florida-only document, combining a living will and health care power of attorney in addition to addressing matters of comfort care and spirituality.

A. A national version of Five Wishes was introduced in 1998. It was originally distributed with support from a grant by the Robert Wood Johnson Foundation.

B. An online version called Five Wishes Online was introduced in April 2011, allowing users to complete the document using an online interface or print out a blank version to complete by hand.

a) Wish 1: The Person I Want to Make Care Decisions for Me When I Can't

b) Wish 2: The Kind of Medical Treatment I Want or Don't Want c) Wish 3: How Comfortable I Want to Be

d) Wish 4: How I Want People to Treat Me

e) Wish 5: What I Want My Loved Ones to Know

Be aware of, and carry out all signature and witness requirements. Determine where these documents will be readily assessable.

a. Advance Care Planning Document

i. Readily accessible to all staff, and health care proxy

b. Advance Directive

i. At home: Post on refrigerator where document can be found

ii. In facility: Taped to inside cabinet of individual's kitchen or bathroom for quick access, First page in medical record, Emergency binder at general nursing station.

\section{Conclusion}

Advance care planning is a process of developing a relevant expression of wishes rather than a single consultation or the signing of a legal document. It is an informed consent, and once completed, is a decision-making document that becomes part of a continuing engagement with the individual, caregiver, and health care provider. The goal of this best practice is to facilitate palliative care advance care planning as part of the individual's care across the continuum of care, especially for those with chronic medical conditions. Included in this continuum of care is acute care, postacute care, assisted living, skilled nursing, and home care.

An informational brochure accompanies this document and is an example of providing individuals the information they need to begin the conversation of advanced care planning with their medical provider/health care representative. The health care provider trained in the ability to communicate will be able to assist the seriously ill individual and/or their representative toarticulate their values and goals of treatment. Inviting the health care proxy to be present at the time of this discussion is encouraged, so that they will also have a clear understanding of their loved one's wishes. "Being Mortal" by Atul Gwande is highly recommended reading as an example of the importance of communicating how we want to be cared for at end of life.

\section{References}

1. Parker SG (1998) "Five Wishes:" An Easy Advance Directive Promotes Dialogue on End-of-Life Care, Robert Wood Foundation, New Jersey, USA.

2. Davies, Frank (1998) "Living Will from Florida Goes Nationwide", The Miami Herald, Miami.

3. David Barile (2011) Director of New Jersey Goals of Care-Town Topics, New Jersey, USA.

4. Having the Conversation, New Jersey Health Care Quality Institute, New Jersey, USA. 


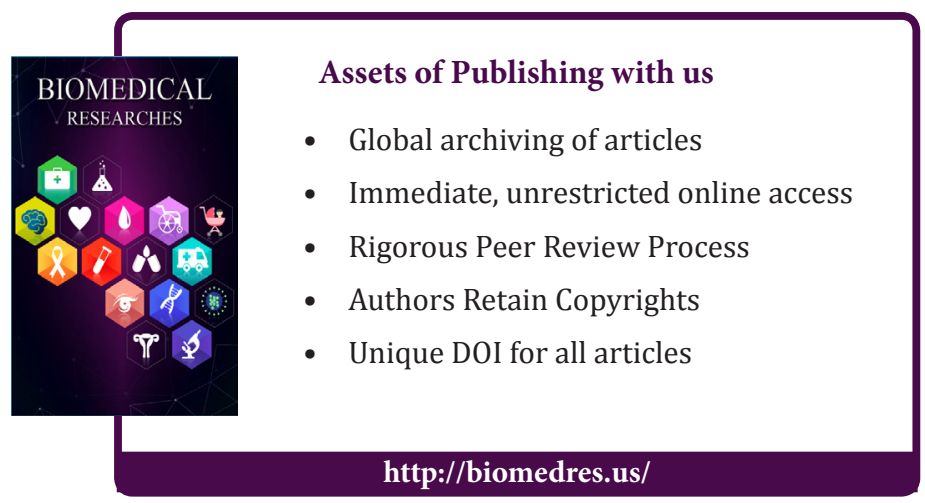

